\title{
Study of the nozzle check valve with respect to its operating characteristics
}

\author{
Roman Klas ${ }^{1, *}$, Vladimír Habán ${ }^{1}$, and Pavel Rudolf ${ }^{1}$ \\ ${ }^{1}$ Brno University of Technology, Faculty of Mechanical Engineering, Victor Kaplan Department of Fluid Engineering, Technická 2 , \\ 61669 Brno, Czech Republic
}

\begin{abstract}
Several modifications were developed when designing the nozzle valve. This study offers an assessment of the properties of new modifications of the nozzle valve design. The main operating characteristics, such as loss and flow coefficients, were determined using a CFD methods. Besides mentioned coefficients, the forces acting on the valve disc are also decisive for the behavior of the valve, both in its steady and unsteady operation. It is important to examine the possible simplification and matching of CFD simulation results from 2D and 3D geometries in terms of subsequent dynamic analysis of the valve. This will be taken into consideration by comparing the above-mentioned operating characteristics, by analyzing the forces acting on the valve disc and comparing the velocity and pressure fields.
\end{abstract}

\section{Introduction}

The presented work proceeds on the development and study of check valves and swing check valves. The construction of these valves, as well as the ways of their application, differs considerably. Of course, this is due, among other things, to the different parameters of the pipe system, in which these fittings are used. From a basic division to low and high pressure hydraulics, we will deal with a low pressure hydraulics. The currently examined check valve is described as the nozzle check valve. As it has already been mentioned, it is a newly developed product based on a well-known principle. In a broader context, the goal is an optimal hydraulic design of check valves. A similar reasearch is also discussed in $[1,2]$. Presented data obtained mainly from CFD simulations served as a support for follow-up experiments on real device. This approach has also been chosen in order to explore some of the phenomena in more detail, e.g. fully visualize flow in the valve, and examine physical quantities that would be difficult to obtain from the experiment.

We are usually interested in the operation of the check valve in terms of the dynamic effect of the whole pipeline. Therefore, it is difficult to optimally design a valve to perform as well as possible under all conditions and without generation of other adverse phenomena. Of course, the problematic operation of the valve is its closure, which depending on the other parameters of the pipeline, leads to a water hammer. The water hammer is pressure wave, which, depending on its frequency, can be transmitted undesirably to the sensitive parts of the pipeline system. Connected sensors or controls for valves are usually affected.

\section{CFD methods and description of the valve}

The static and some dynamic valve characteristics were evaluated using CFD simulations. Velocity and pressure fields were also monitored and the ANSYS 17.2 ANSYS Fluent was used as a basic tool for the analysis. The valve geometry was considered both as two and three dimensional and the computational mesh was created in ANSYS Meshing. More information can be found in Table 1.

Table 1. Description of numerical model.

\begin{tabular}{|l|c|c|}
\hline & \multicolumn{1}{|c|}{$2 \mathrm{D}$} & \multicolumn{2}{c|}{$3 \mathrm{D}$} \\
\hline Pipeline diameter & \multicolumn{2}{|c|}{ DN100 } \\
\hline $\begin{array}{l}\text { Number of } \\
\text { computational cells }\end{array}$ & \multicolumn{1}{|c|}{400000} & $\begin{array}{c}\text { 9-16 } \\
\text { millions }\end{array}$ \\
\hline $\begin{array}{l}\text { Turbulence model } \\
\text { and near wall } \\
\text { modeling }\end{array}$ & $\begin{array}{l}\text { realizable } \mathrm{k}-\varepsilon \\
\text { non equilibrium wall function }\end{array}$ \\
\cline { 2 - 3 } $\begin{array}{l}\text { realizable } \mathrm{k}-\varepsilon \\
\text { scalable wall function }\end{array}$ \\
\hline Boundary conditions & $\begin{array}{l}\text { Inlet: velocity inlet } \\
\text { Outlet: pressure outlet }\end{array}$ \\
\hline Calculation mode & $\begin{array}{l}\text { unsteady, incompressible } \\
\text { flow }\end{array}$ \\
\hline
\end{tabular}

Two methods of near-wall treatment were chosen in the test tasks, since the size of the Reynolds number values ranged from 0 to $10^{6}$ and the computational mesh was built in terms of its quality and the way of modeling

\footnotetext{
* Corresponding author: klas@,fme.vutbr.cz
} 
the boundary layer regions. More can be found, for example, in [3-7]. Of course, wall functions have been chosen with respect to the smallest number of computational mesh cells and the lowest computational requirements. The RNG $\mathrm{k}-\varepsilon$ turbulence model is recommended in the literature with regard to the higher accuracy of CFD simulations with respect to experimental data $[8,9]$. However, using the RNG $\mathrm{k}-\varepsilon$ turbulence model settings in this case provided a worse agreement between CFD simulations results and the experimental data than the realizable $\mathrm{k}-\varepsilon$ model. Hydraulic valve losses in the case of RNG settings have been underestimated.

The nozzle check valve excels by relatively small installation dimensions. The valve can also be described as approximately axisymmetric. This, of course, can help to improve the accuracy of a simpler two-dimensional analysis. Of course, this may help to improve the accuracy of a simpler two-dimensional analysis. However, the properties of the devices that are adapted to the experimental testing and are different in some aspects from the production version of the valve, are problematic, see Fig.1.

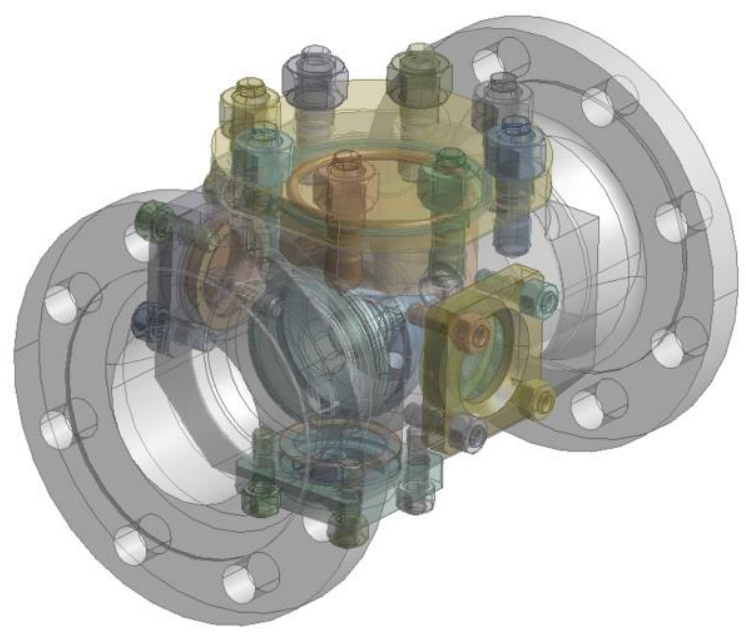

Fig. 1. The nozzle check valve, 3D P simulation.

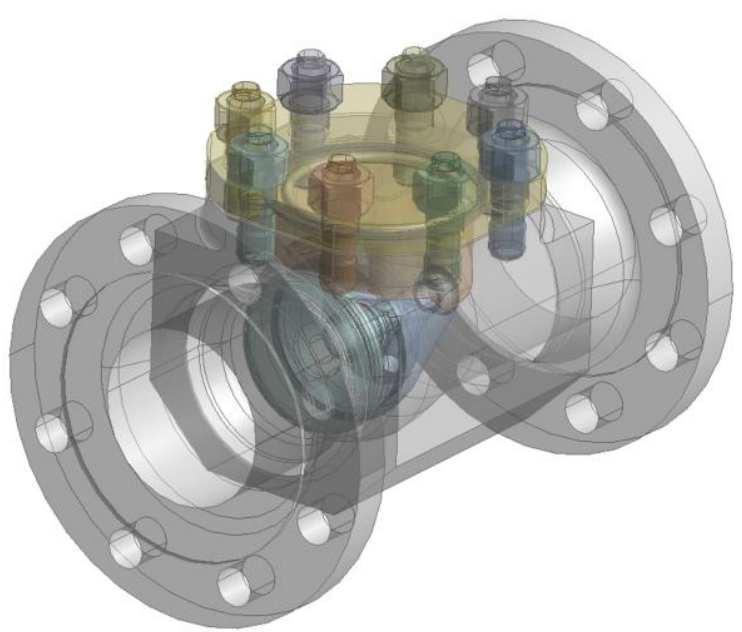

Fig. 2. The nozzle check valve without windows, 3D simulation.

As can be seen from the Fig. 1, the device was completed with several windows to ensure the visualization of the velocity field. The mounting hole at the top of the valve is fitted with an insert. The hole may also serve as an observation window if the insert is removed. Two 3D models were created due to observation windows, see Fig.2. The windows are omitted in the second 3D model the. Therefore, the number of computational cells in the three-dimensional model in Tab. 1 is quite different.

The CFD simulation task will, among other things, decide the difference in the results of the experimental device (Fig.1) and the nozzle check valve, which is very close to the current production version. A view of the interior of the valve is provided in Fig.3.

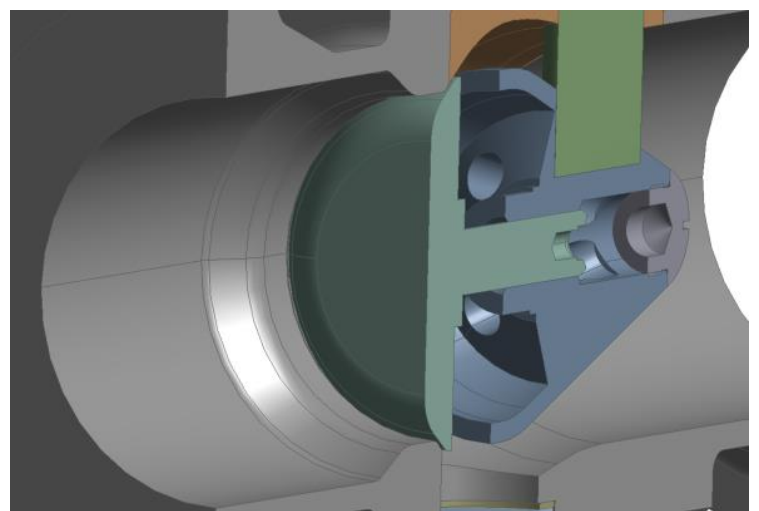

Fig. 3. Interior of the nozzle check valve, 3D simulation.

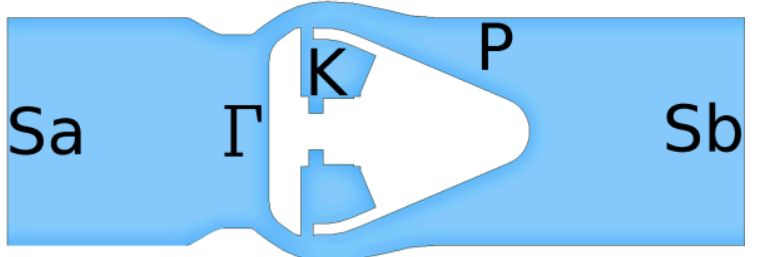

Fig. 4. Interior of the nozzle check valve, 2D simulation.

Fig. 4 shows the model used in 2D simulation. This simulation was solved as axisymmetric. Of course, the pipeline parts of the $2 \mathrm{D}$ and $3 \mathrm{D}$ simulations were also considered to eliminate the effect of the boundary conditions on the interior of the valve. The influence of boundary conditions is discussed in more detail e.g. in $[10,11]$. The disc itself was also tested in the valve chamber in addition to the above-mentioned simulations (Fig.5). The reason is the future study of the dynamic behavior of the valve and decsription of the force magnitude from the fluid acting on the disc.

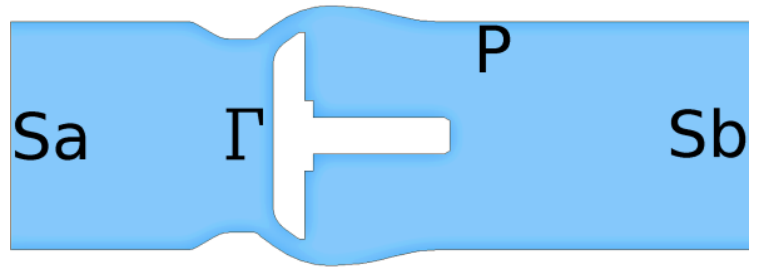

Fig. 5. The disc in the valve interior, 2D simulation.

\section{Static characteristics}

The valve was tested in operating modes that were defined by mass flow. Tab. 2 provides informations about the 
mean values of absolute velocities in the inlet section of the valve and the sizes of its opening. Values in the columns do not necessarily match each other.

Table 2. Range of mean absolute velocities in the valve and the valve opening sizes.

\begin{tabular}{|c|c|c|c|c|c|c|c|c|c|c|}
\hline $\mathrm{v}\left[\mathrm{m} \cdot \mathrm{s}^{-1}\right]$ & -3 & -2 & -1 & 1 & 2 & 4 & 6 & 8 & 10 & 10.5 \\
\hline $\mathrm{z}[\mathrm{mm}]$ & & & & & 2 & 5 & 7 & 10 & 15 & 20.5 \\
\hline
\end{tabular}

The additional symbols used in the following figures for the pressure and velocity fields in the valve are shown in Table 3.

Table 3. The additional list of symbols.

\begin{tabular}{|c|l|}
\hline $\mathrm{N}$ & non equilibrium wall function \\
\hline $\mathrm{ON}$ & disc only \\
\hline $\mathrm{P}$ & windows in the valve walls \\
\hline $\mathrm{S}$ & scalable wall function \\
\hline $\mathrm{SM}$ & $\begin{array}{l}\text { non-friction valve walls, disc with } \\
\text { friction }\end{array}$ \\
\hline $\mathrm{Sa}-\mathrm{Sb}$ & $\begin{array}{l}\text { the difference between the inlet and } \\
\text { outlet sections of the valve }\end{array}$ \\
\hline Inlet - Outlet & $\begin{array}{l}\text { the difference between surfaces } \\
\text { with boundary conditions }\end{array}$ \\
\hline
\end{tabular}

Since the static characteristics of the valve will be presented, the flow in the valve was simulated for several basic positions of the disc according to the degree of its opening. Convergence of CFD simultions, especially 3D ones, was relatively weak due to high valve velocities with small valve openings and high flow rates. For this reason, it was not possible to realize all the simulations in all modes corresponding to Tab. 2. Of course, many of them do not match any of the operating modes.

The dependence of static pressure difference on flow rate is one of the most important characteristics that describe the static behavior of the valve. For the time being, the dependencies corresponding to the valve opening $z=7 \mathrm{~mm}$ will only be shown, see Fig. 6 and Fig. 7. The results of $2 \mathrm{D}$ and $3 \mathrm{D}$ simulations as well as the choice of different wall functions for three-dimensional problems are compared. Preliminary, we can say that non equilibrium wall functions will be preferred. Just 2D simulations should bring simplification of further valve analysis, as already mentioned.

The evaluations of the flow parametres on the different boundary surfaces are also presented, see Tab. 3 . It is not possible to trust the accuracy of static pressure evaluation on Inlet - Outlet surfaces. At the same time, the differencies in the areas of $\mathrm{Sa}-\mathrm{Sb}$ due to the boundary conditions are shown. Of course, it is paramount that the turbulent or laminar velocity profile was fully developed before the computational domain of the valve interior itself. The above mentioned is also partly related to nonmatching positions of pressure sensors in CFD and experiment. More details on agreement of numerical and experimental approach can be found in [12] for example. Of course, the location of the sensors does not completely match the input and output surfaces to the valve. The hydraulic losses produced by the valve are relatively small, and the inclusion of a part of the pipeline into the evaluated section may produce an undesirable error or misrepresentation.

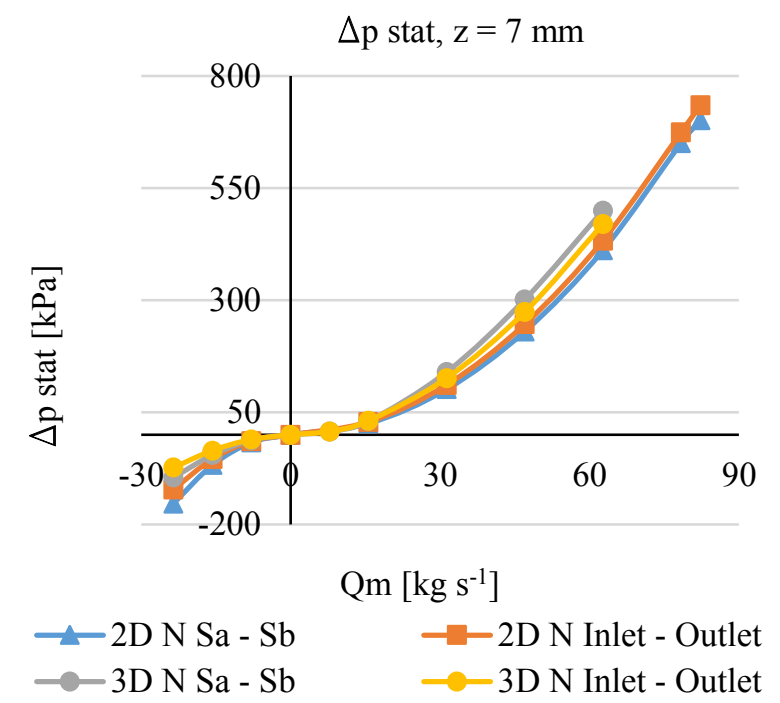

Fig. 6. Downstream and upstream static pressure differencies.

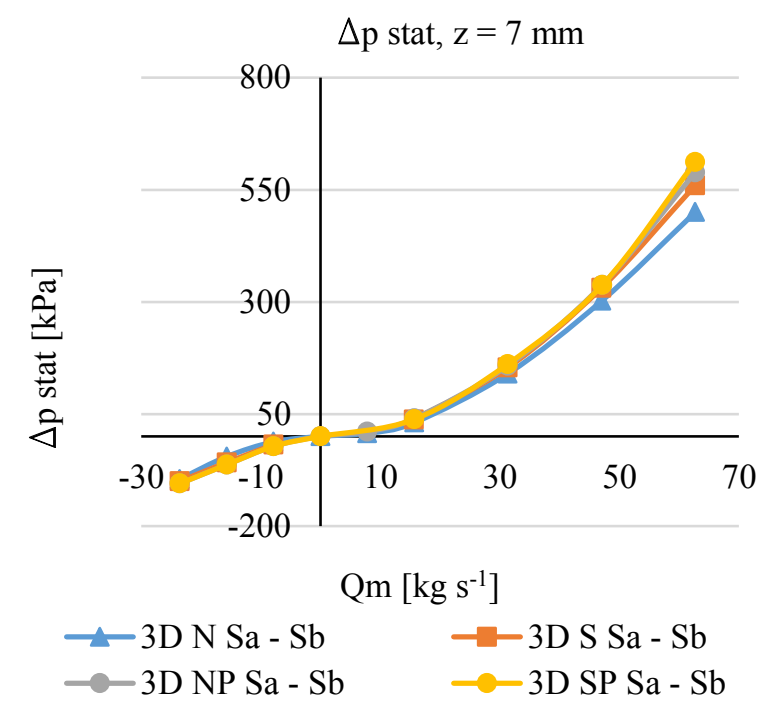

Fig. 7. Downstream and upstream static pressure differencies.

It is evident from Fig. 6 that there are some differences between 2D and 3D simulations. These differences mainly correspond to the areas on which the boundary conditions are located. On the other hand, the agreement of static pressure differencies is very good on the surfaces corresponding to the inlet and outlet of the valve. The following igure (Fig. 7) offers a comparison of 3D valves with and without windows (Fig.1 and Fig.2) as well as comparison of the use of two different wall functions, see Tab.1 and Tab.3. It seems that scalable wall functions predict a more pronounced pressure loss. The greater static pressure differences of the valve with the windows was foreseeable.

It is also evident that the pressure difference was also evaluated into the backflow region, considering that the backflow through the valve may theoretically occur. This condition is undesirable from the water hammer pressure extreme point of view. The flow coefficients $\boldsymbol{K} \boldsymbol{v}$ as defined in (1) are plotted in the following Figures 8 and 9. 
The flow coefficients Kv standardly defined by (1) are captured in the following Figs. 8 and 9.

$$
K_{V}=Q \sqrt{\frac{\Delta p_{r e f} \rho}{\Delta p \rho_{r e f}}}
$$

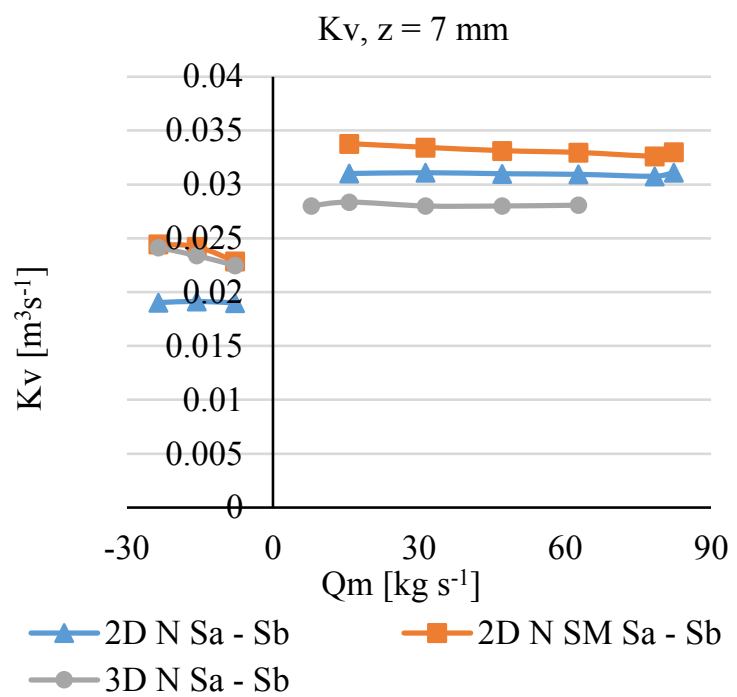

Fig. 8. Downstream and upstream flow coefficients.

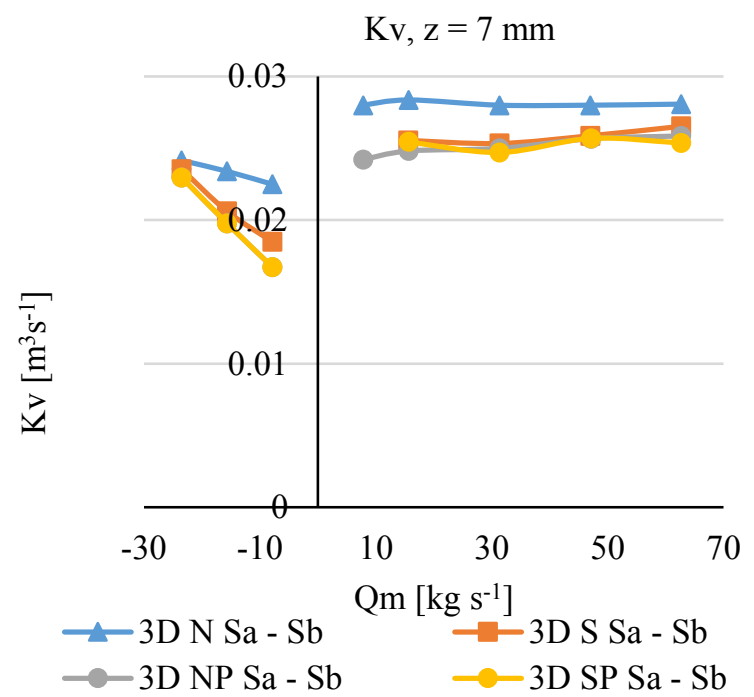

Fig. 9. Downstream and upstream flow coefficients.

It results from Fig. 8 that the highest values of the flow coefficient are achieved by a valve whose walls are defined as friction-free walls. The reason for including this simulation in the attached statistics is to analyze the force acting on the valve disc. Furthermore, it is clear from Fig. 9 that the flow coefficient $\mathrm{Kv}$ is slightly higher even for the 3D simulation of the valve without windows. However, the differences are not large. The choice of different wall functions was most evident in the model without windows (Fig.2).

Another pair of graphs are Fig.10 and Fig.11, which capture the magnitude of the loss coefficient according to the mass flow through the valve (2).

$$
\xi=\frac{\Delta p}{\rho \frac{v^{2}}{2}}
$$

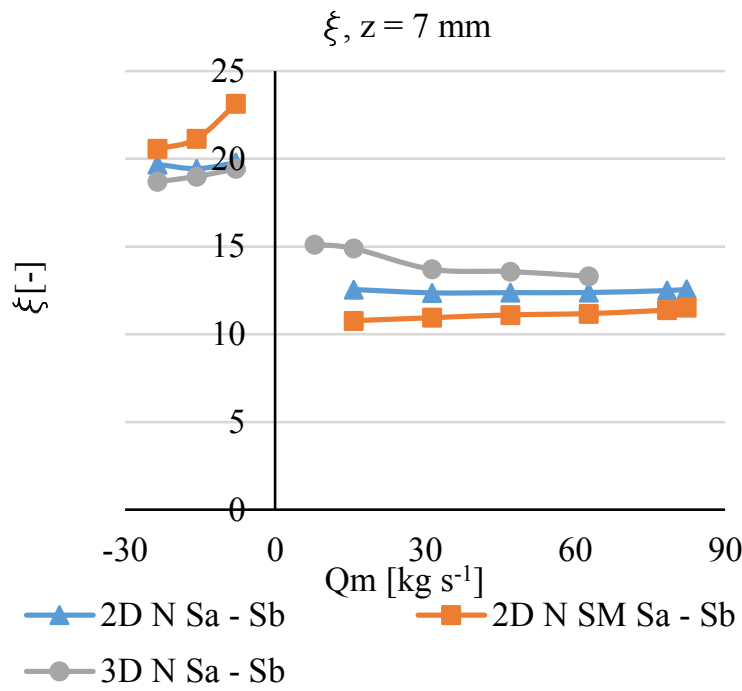

Fig. 10. Loss coefficient.

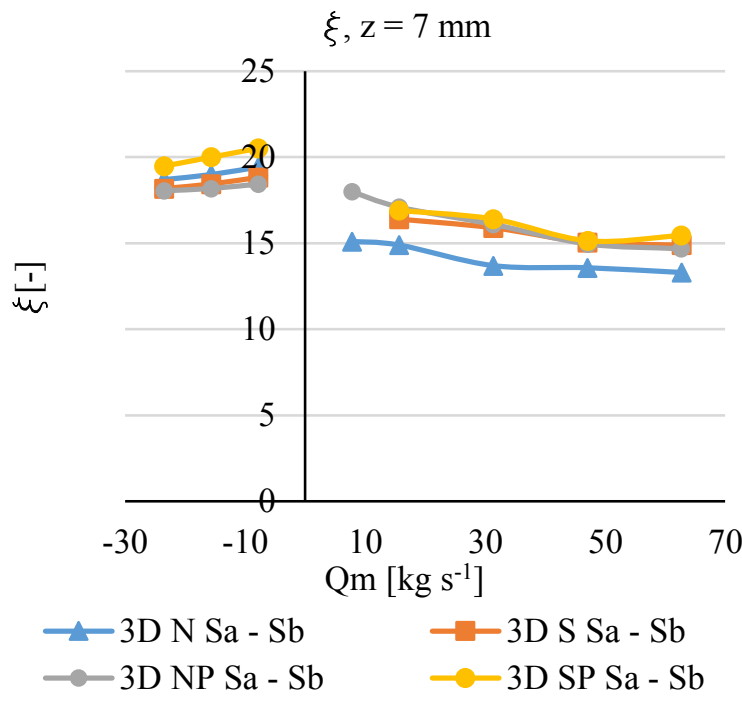

Fig. 11. Loss coefficient.

The loss coefficient increases with a decreasing flow rate in accordance with the assumptions. Slightly greater difference between 2D N and 3D N simulations for $\boldsymbol{z}=7$ $\mathrm{mm}$ takes into consideration the effect of the fin on which the valve disc bushing is located, see Fig. Fig.3. The windows in the valve wall increase the deviation from the axisymmetric flow, Fig.11. There is a very small difference between $3 \mathrm{D} \mathrm{S}$ and 3D SP simulations for downstream flow rates. Such situation is inconsistent with expectations. Therefor the $\mathrm{N}$ model will be preferred, Tab.3.

It will be important for the dynamic behavior of the valve to determine the magnitude of the force by which fluid acts on the valve disc. The forces Fx3 acting on the axis of the valve, depending on the mean value of the static pressure, are shown in Fig. 12 and Fig. 13. 
Note: No force from the spring is taken into consideration in any of the graphs. Positive effect is assigned to the force acting in the direction of downstream flow through the valve.

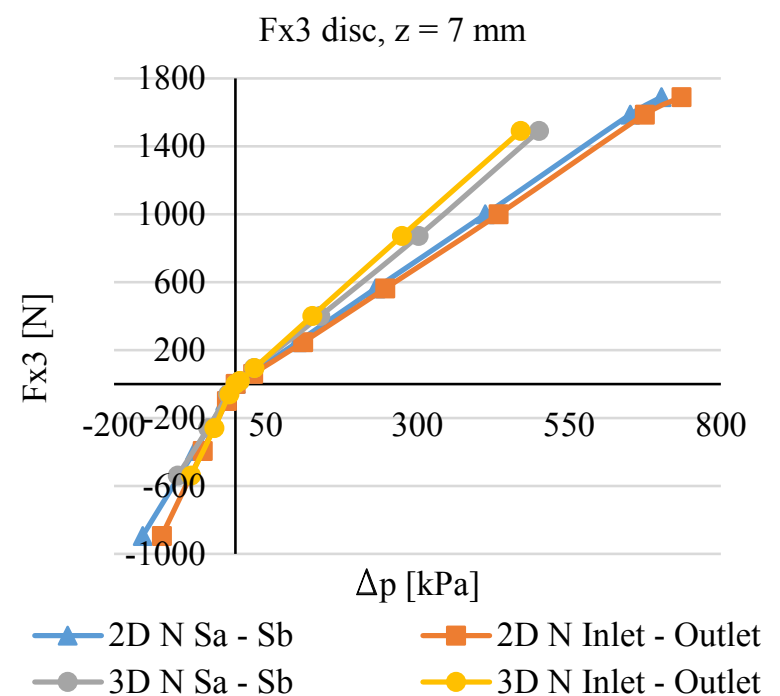

Fig. 12. The forces acting on the valve disc.

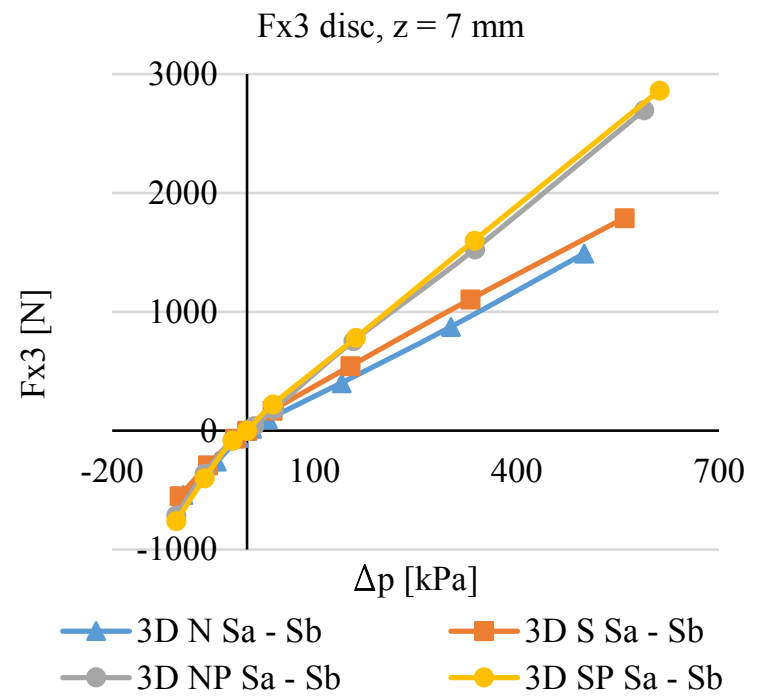

Fig. 13. The forces acting on the valve disc.

All forces are almost linear. Conformity of 2D and 3D models is generally better negative mass floe rate values or static pressure differences. The magnitude of the valve forces with or without windows do not differ for both cases of selected wall functions, which is convenient. This allows more usability of the already obtained data and also presents a lesser risk of choosing unsuitable wall functions. It can also be said that the force on the disc is higher than in case of the smooth shape of the valve with the windows. This may have an impact on a visualization experiment that verifies the dynamic behavior of the valve, which should be realized just on the valve with the windows (Fig.1).

Valve force acting on the immobile solid wall of the valve was also evaluated in addition to the force acting on the plug (excluding the inlet and outlet pipes). The ratio of these forces will be interesting from this point of view.
Force analysis can be done by integrating the NavierStokes equation (3) within the valve interior.

$$
\rho_{K}\left(\frac{\partial v_{i}}{\partial t}+\frac{\partial v_{i}}{\partial x_{j}} v_{j}\right)=\rho_{K} g_{i}-\frac{\partial p}{\partial x_{i}}+\frac{\partial \Pi_{j i}}{\partial x_{j}}
$$

Obviously, it would be problematic to integrate all the terms in equation (3) without CFD simulations. This fact makes it more difficult to build a corresponding onedimensional model that predicts the behavior of the valve in the piping system. Even with knowledge of CFD simulation data, it is not easy to simply substitute a term in (3) in which includes the irreversible stress tensor $\boldsymbol{\Pi}$. Of course, the force from the fluid affects the fixed and moving walls of the valve. We can assume at least approximately an axisymmetric static pressure $\boldsymbol{p}$ distribution when determining the resultant force effect. However, such simplification will not be useful for shear stresses. The adhesion of liquid to the walls (no slip boundary condition) can only be considered. The fluid force on the valve disc $\boldsymbol{F}_{\boldsymbol{K}}$ can then be expressed, for example, by equation (4).

$$
\begin{gathered}
F_{K}=\rho_{K} \ddot{x}_{3} V_{\Gamma}-\rho_{K}\left(x_{3 S a}+x_{3 S b}\right) \frac{\partial Q}{\partial t}+R_{a b} Q^{2} S \\
R_{a b}=\frac{\xi}{2 S^{2}}
\end{gathered}
$$

The hydraulic resistance $\boldsymbol{R}_{\boldsymbol{a} \boldsymbol{b}}$ (5) then represents the term in (3), which describes the magnitudes and distribution of static pressures and shear stresses on the solid walls. The normal and tangential forces acting on the moving valve disc are represented by $\boldsymbol{F}_{\boldsymbol{K}}$. However, it follows from this consideration that $\boldsymbol{R}_{\boldsymbol{a}} \boldsymbol{b}$ can not be determined from the total loss coefficient in the valve (eg experimental data), because it includes the hydraulic losses caused by the disc itself.

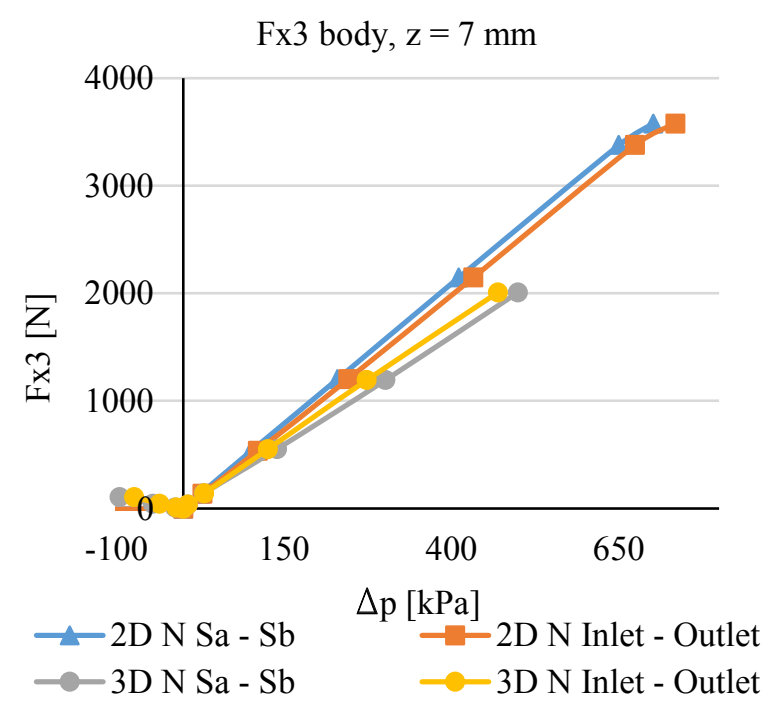

Fig. 14. The forces acting on the valve body.

For these reasons, it is important to get an idea of the ratio of forces only acting on the valve disc and on its fixed solid walls, respectively, of the hydraulic losses 
caused by the disc and the non-moving solid walls of the valve. Although it is necessary to consider mutual interaction of that the pressure and velocity fields around the disc and around the solid walls. Equation (4) also indicates that it is necessary to focus attention on the unsteady flow rate diferencies that could significantly affect $\boldsymbol{F}_{\boldsymbol{K}}$. However, the magnitudes of forces on the whole body of the valve will be presented first, Fig. 14 and Fig. 15.

Fig. 14 shows that the similarity of $2 \mathrm{D}$ and $3 \mathrm{D}$ simulations is sufficient. Also, the forces determined by the negative size of the static pressure difference are important, i.e., during backflow of the fluid through the valve. In some cases, the magnitude of these forces may be positive and not negative, as in Fig. 14. Obviously, the orientation of the force vector is given by the shape of the valve, the fin and the guide bushing. Some body of guide bushings are also designed as through-flow channel. Even in the case of evaluation of the axial force on the valve wall, there is a good consistency between two different wall functions (Fig.15). The forces also maintain a linear dependence on the pressure difference in the valve.

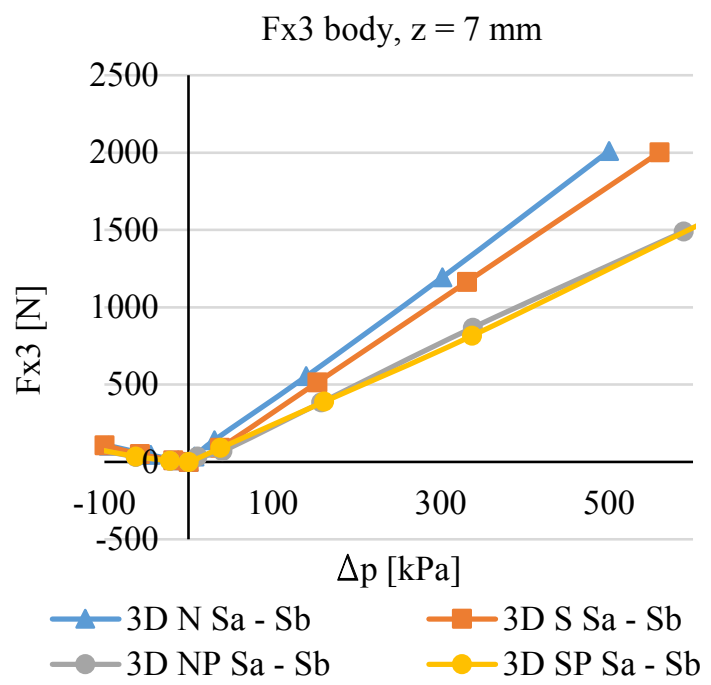

Fig. 15. The forces acting on the valve body.

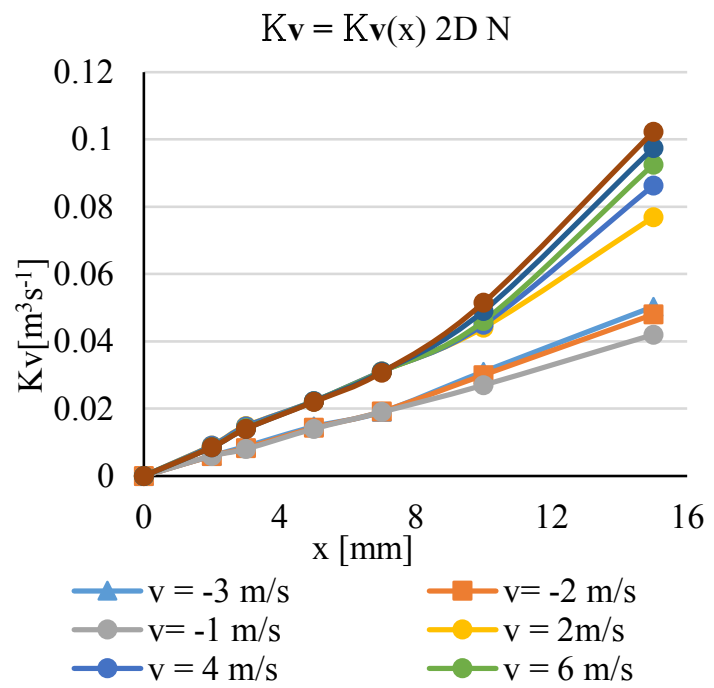

Fig. 16. Flow coefficient.
All previously presented characteristics corresponded to one position of the valve disc. The aim was to specify the typical characters of the physical quantities. In addition, the static characteristics will be shown for the different positions of the disc $z$. Fig. 16 shows the flow coefficient $\boldsymbol{K}_{\boldsymbol{v}}$ in case of simulation 2D N (Tab.3).

It is clear that the curves are divided into two basic parts according to the direction of flow. The obtained data are not easily separable due to the number of curves corresponding to the velocities in Tab. 2. The lower curves correspond to backflow.

Similarly, loss coefficients $\xi$ for 2D N simulation are shown, see Fig. 17. It is clear from this figure that there is a clearer separation of downstream flow and backflow. And it is also obvious that the backflow velocities almost correspond to the highest loss coefficients $\xi$ in range of disc position $\boldsymbol{z}$.

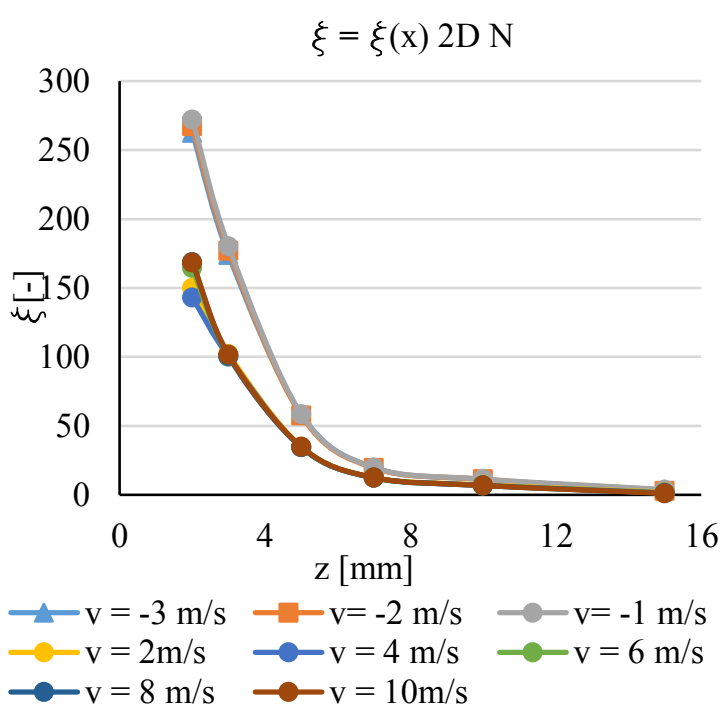

Fig. 17. Loss coefficient.

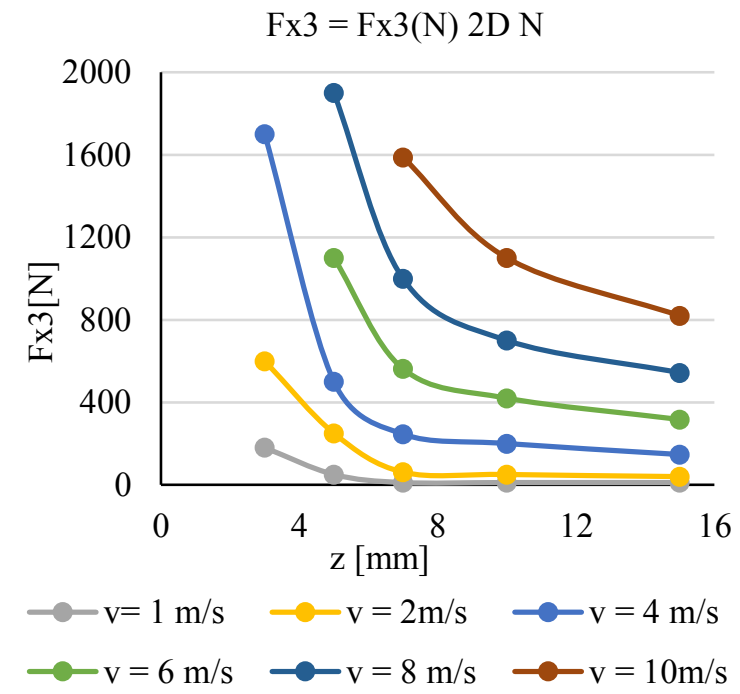

Fig. 18. The forces acting on the valve disc.

The force magnitude for the 2D $\mathrm{N}$ simulation are shown in Fig.18. Each curve corresponds to approximately hyperboles and should increase theoretically for $z=0$ to infinity. However in the case of 
a real valve the magnitude of the force acting on the disc corresponds to the spring preload force, for $z=0$.

Undoubtedly, a crucial point of view for assessing static characteristics will be their comparison with the experimental data. Experimental data were available for valve without windows (Fig. 2) at the time of writing this paper. Of course, the valve disc is moved when the flow is changed, so its position, which can be associated with the flow rate, needs to be known. Flow and loss coefficients were evaluated for constant flow during the experiment. The problem with the unknown valve disc position has been resolved through CFD simulations. Software ANSYS 17.2 allows 1DOF (degree of freedom) simulation of valve disc movement. Therefore, the dynamic simulation has been realized for the constant flow rate, for which the valve disc has found its operating position. The dependence of the loss and flow coefficients was then obtained by interpolation of the static characteristics in dependence on the flow rate (Fig. 19 and Fig. 20).

The valve was tested on the closed test circuit. Circuit consists of the connecting pipes, pressurized vessel, which is connected to vacuum pump (for cavitation testing), the tested valve itself and feeding pump. Flow rate was measured using induction flowmeter, pressure difference between valve inlet and outlet was assessed using pressure sensors. Measurement procedure and data evaluation is according to valid standard ČSN EN ISO 9906.

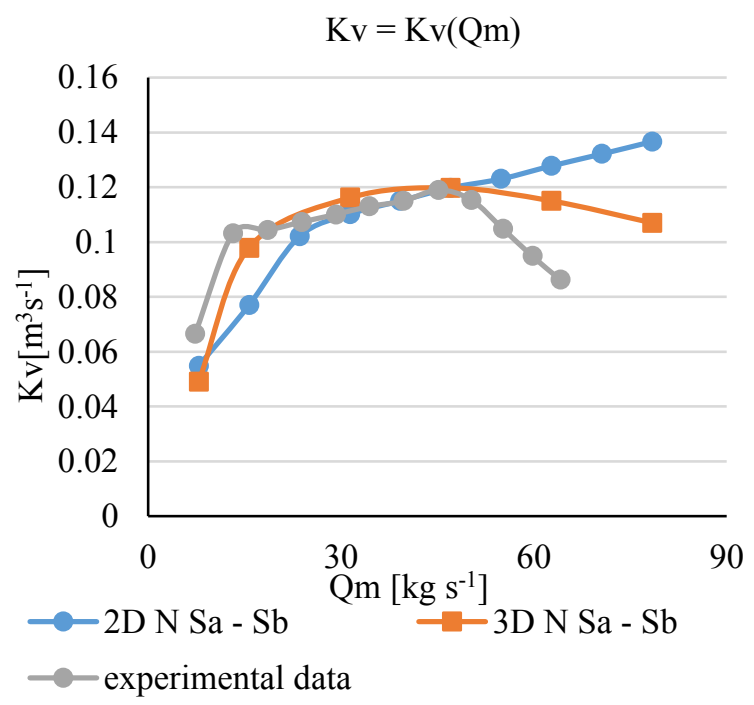

Fig. 19. Flow coefficient.

Agreement of CFD simulation with experiment is good for medium operating flow rates in both cases. The difference in results is most noticeable in 2D simulations in the high flow rate area. The loss coefficient usually decreases with increasing flow rate and the flow coefficient increases. However, there are coefficient break points for this valve and both coefficients manifest nonstandard characteristics. The reason is the embedded fin in $3 \mathrm{D}$, the cavitation and the separated and swirling flow around the inner parts of the valve.

Since the magnitude of the loss and flow coefficient in 3D simulations shows differences in comparison with the experiment, the dependencies of these coefficients for the maximum opening of the valve are also shown, namely $z$ $=20.5 \mathrm{~mm}$, Fig. 21 and Fig. 22. However, it should be noted that the valve may not be fully open in normal operating mode. Trend of coefficients from 3D simulations and experiment occurs with increasing valve opening approximately from $z=10 \mathrm{~mm}$ but first very slightly (Fig. 21 and Fig. 22). This disc position corresponds to half of the maximum lift of the disc.

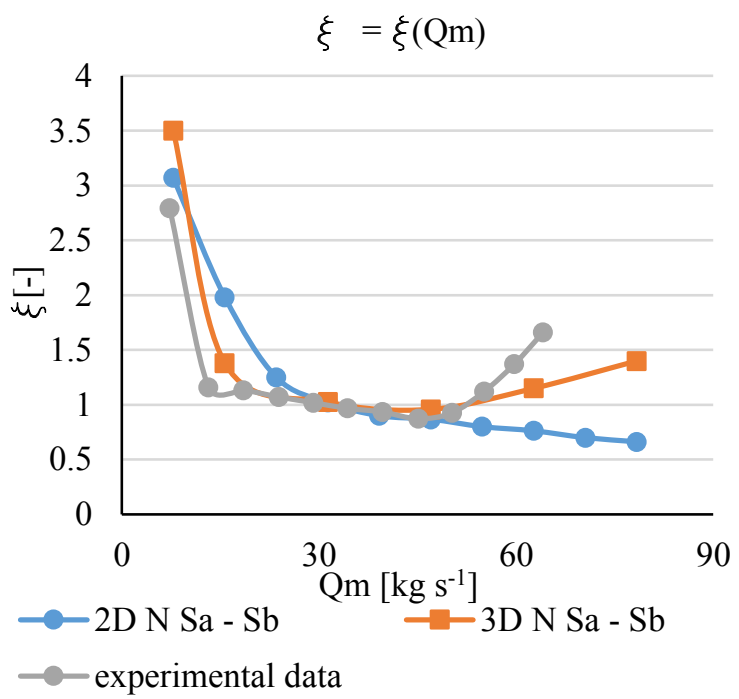

Fig. 20. Loss coefficient.

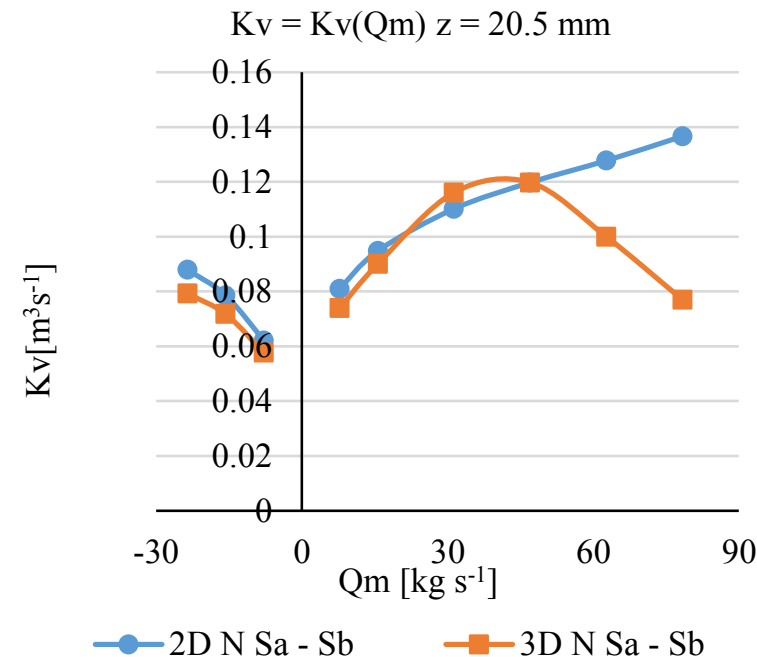

Fig. 21. Flow coefficient

Valve characteristics with constant disc position and variable flow rate are the last dependencies to be provided. A closer study of the mathematical model of force acting on the valve disc (4) is the reason for their presentation. An idea of the actual magnitude of the unsteady term in (4) will be built upon them. The magnitude of the unsteady term in the force equation was determined first. This magnitude was then compared as a force increment with the increase in force in the unsteady change of flow in CFD simulation. The change in test flow was linear and ranged from positive to negative for the time corresponding to the closing time $\boldsymbol{T} \boldsymbol{s}$ of the valve. 
The $\boldsymbol{T} \boldsymbol{s}$ values are described in the corresponding curve legend in the graph. The dependencies of disc forces on the static pressure difference were again linear. It seems that, due to different sizes $\Delta \boldsymbol{p}$, the magnitude of these forces differs considerably. For this reason, the forces were plotted according to the constant and variable flow through the valve, see Fig. 23.

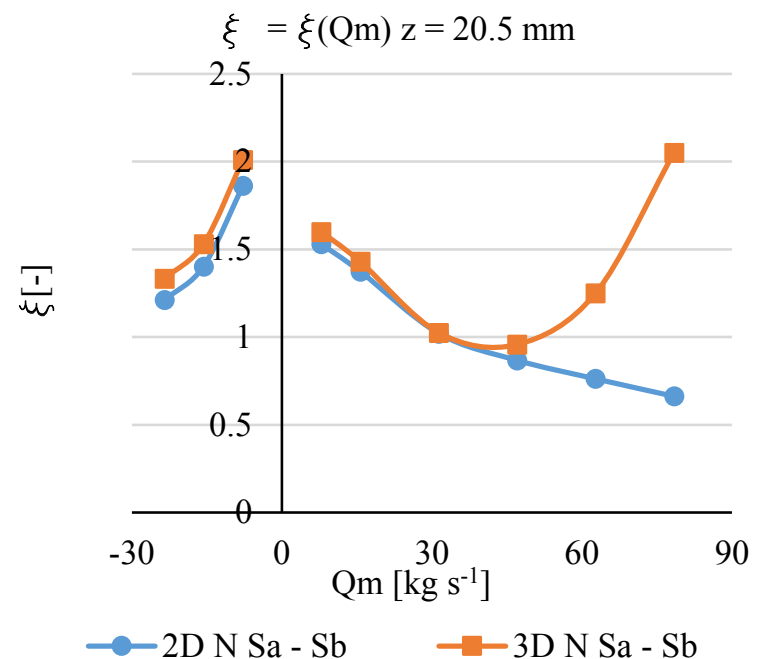

Fig. 22. Loss coefficient.

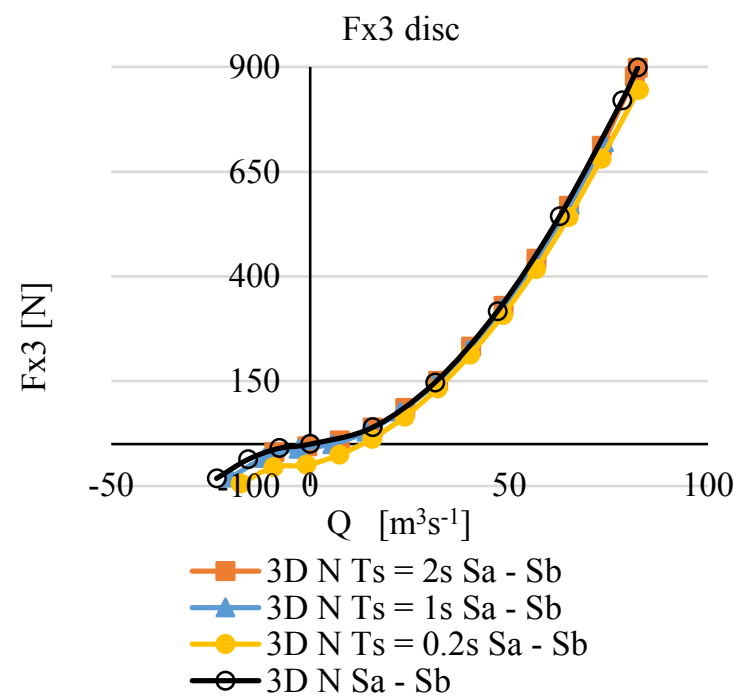

Fig. 23. The forces acting on the valve disc.

Valve shut-off time $\boldsymbol{T s}=0.2 \mathrm{~s}$ is extreme and can only be realized in case of valve bypass or pipe failure. Fig. 24 shows the ratio of the force gains on the disc versus the stationary state in the CFD simulations and in prediction from the analytical model of force (4). The ratio of the force gains is more balanced when the valve closes faster. The model of the unsteady force component in (4) assumes a constant distribution of local acceleration on the $\mathrm{Sa}$ and $\mathrm{Sb}$ surfaces, respectively, considers its mean value according to the flow rate. A shorter valve closing time achieves a more balanced change of the absolute velocity vectors on the surfaces. In the case of closing time $\boldsymbol{T} \boldsymbol{s}=0.2 \mathrm{~s}$, the ratio of the analytical model of the unsteady force component $\boldsymbol{F}_{\boldsymbol{x} 3}$ is $40-80 \%$ of the value obtained from the CFD simulation. This ratio increases with increasing time and becomes an extreme whose value has a growing character. On the other hand, the flow rate at which the extreme occurs decreases. The whole problem can also be described as a prediction of an unsteady velocity profile. Therefore, it is more advantageous to extend the valve area to the inlet and outlet pipe sections in the mathematical model so that the velocity profile is not significantly affected by the flow in the valve on the Sa and Sb surfaces. However, those parts of the pipeline would be included in the simulation, which do not actually belong to the valve.

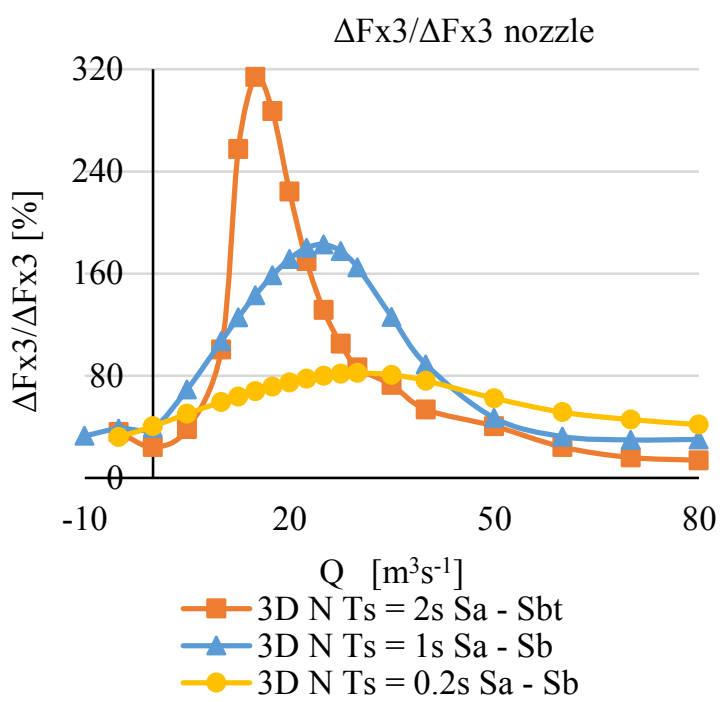

Fig. 24. The ratio of unsteady force components evaluated from analytical approach and from CFD simulations.

\section{Comparison of the velocity and pressure field}

It will be important to compare the flow field in the valve interior to complement the study of the valve geometries. Of course, the main objective is to determine the differences and to assess the possibilities of reducing the computational requirements of CFD simulations. The velocity and pressure fields in two directions of flow will only be monitored. Flow rates correspond to the mean velocity magnitude of the absolute velocity, $v=2 \mathrm{~m} \cdot \mathrm{s}^{-1}$ for Q1 and $v=-1 \mathrm{~m} \cdot \mathrm{s}^{-1}$ for Q2. The flow rate Q1 represents the normal flow through the valve. The other labels used in the figures correspond to the symbols in Tab. 3. The valve opening $z=7 \mathrm{~mm}$ is identical to the position of the disc in the previous static characteristics.

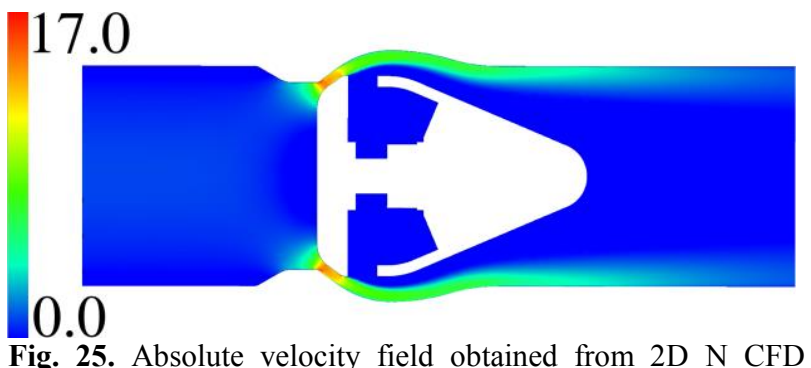

Fig. 25. Absolute velocity field obtained from 2D N CFD simulation, flow rate Q1. 
First, Fig. 25 - 34 present a velocity field. Significant differences are not observable in Fig. 25-28. The embedded fin on which the disc and guide bushing are attached affects the flow in the 3D geometry on Fig. 28. However, the flow is axisymmetric in the remaining parts of the valve, and the magnitudes of the absolute velocities are almost identical in all cases.

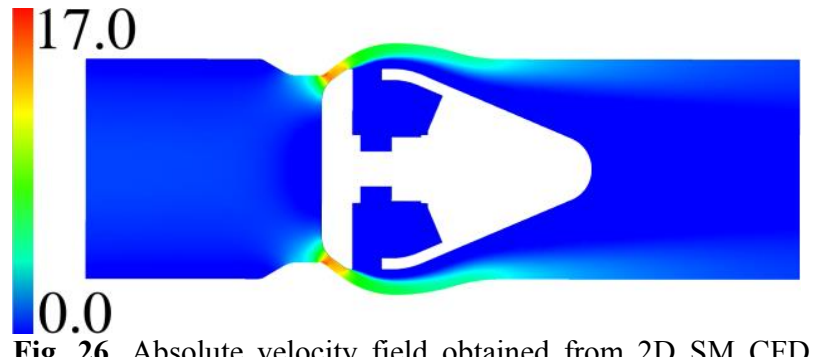

Fig. 26. Absolute velocity field obtained from 2D SM CFD simulation, flow rate Q1.

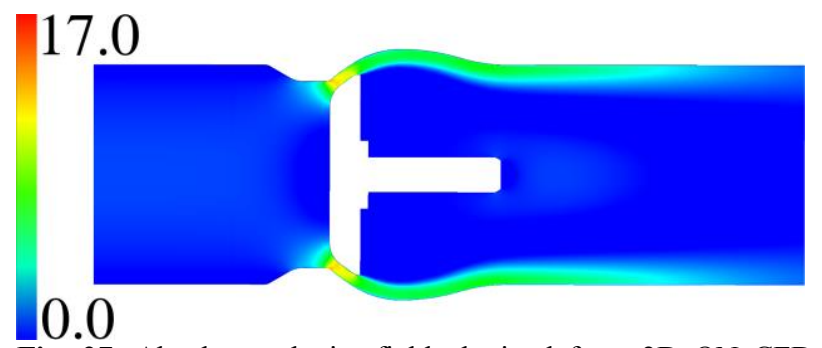

Fig. 27. Absolute velocity field obtained from 2D ON CFD simulation, flow rate $\mathrm{Q} 1$.

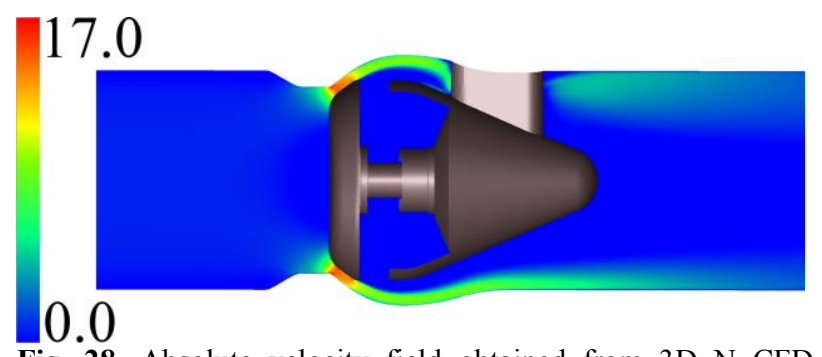

Fig. 28. Absolute velocity field obtained from 3D N CFD simulation, flow rate Q1.

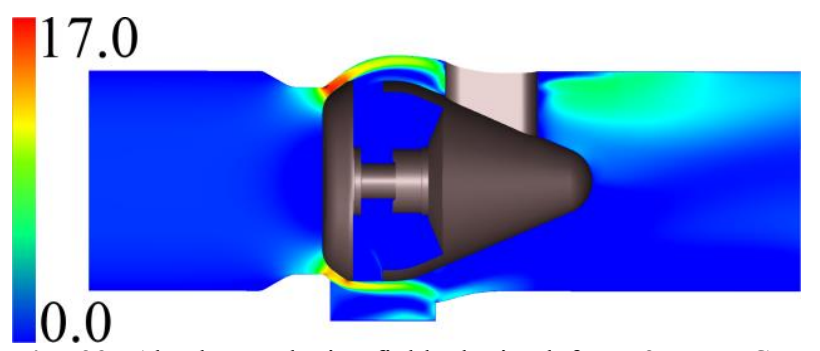

Fig. 29. Absolute velocity field obtained from 3D NP CFD simulation, flow rate Q1.

The valve windows (Fig. 1), which significantly interfere with the flow field in Fig. 29, appear to be problematic. The windows are an element that deforms significantly the flow around the valve disc. The window frames intersect approximately one-third of the disc circumference. However, the situation in the case of backflow through the valve represented by the flow Q2 is significantly different. The flow around the disc and inner parts of the valve in Fig. 30 and Fig. 31 is noticeably different from the flow around the disc itself in Fig. 32 and the 3D geometry in Fig. 33. The computational meshes were very similar in 2D geometries, and the feature that could only have affected the resulting flow character is the axisymmetric condition. Also, the $\mathrm{k}-\omega$ SST turbulence model has been tested for 2D and 3D geometry to be sure in this particular case with respect to the possible flow separation of the 3D model. However, the velocity field did not change its character in any of the cases. The disc itself appears to be the most identical in terms of flow behind the disc in backflow for 2D geometry (Fig.32) and complete 3D geometry for the valve without windows (Fig.33). It should be noted that the high velocity magnitude regions in the figures are relatively short and the fluid on the walls does not slip. It is therefore important for the resulting flow field to reflect the shape of the streamlines between the disc and the guide disc as well as the ratio between the shear and the pressure forces. The whole body of the valve is a slip wall in Fig. 31, and the flow field has the character of an intermediate step between the results of $2 \mathrm{D}$ and $3 \mathrm{D}$ simulations, i.e., Fig. 30 and Fig. 33. Even simulation of the flow around the disc (Fig.32), in which the shear force part due to the absence of the guide disc and bushing is smaller, indicates this.

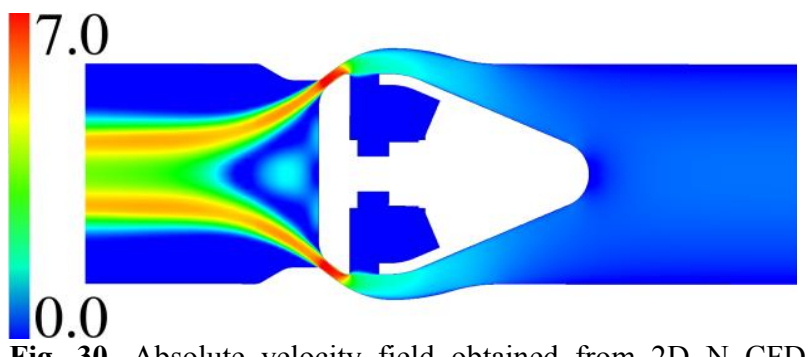

Fig. 30. Absolute velocity field obtained from 2D N CFD simulation, flow rate Q2.

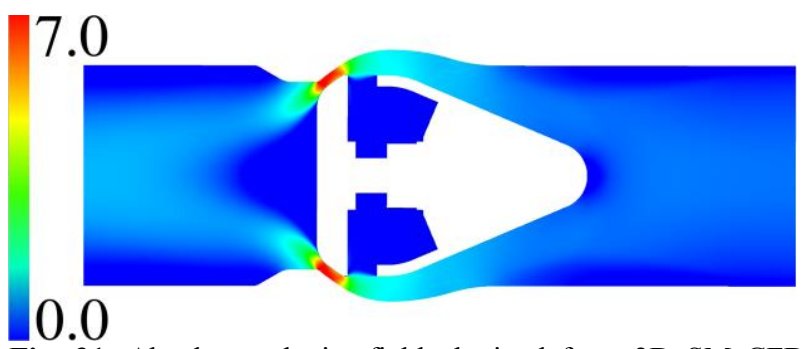

Fig. 31. Absolute velocity field obtained from 2D SM CFD simulation, flow rate Q2.

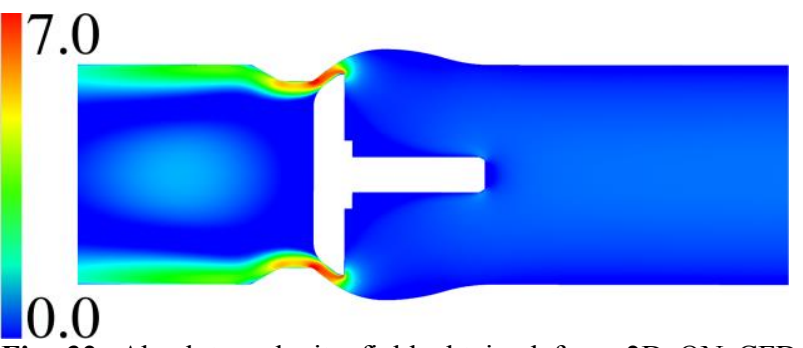

Fig. 32. Absolute velocity field obtained from $2 \mathrm{D}$ ON CFD simulation, flow rate Q2.

As could be expected, the most disordered velocity field is represented by geometry with windows, see Fig. 34. The window frame itself also splits the stream and causes the flow between the disc and the guide disc. These facts may affect the subsequent assessment of the dynamic properties of the valve during the experimental 
testing. Note: The velocity magnitude scale has to be changed for both 3D simulations because the maximum values are slightly higher than for the $2 \mathrm{D}$ geometry.

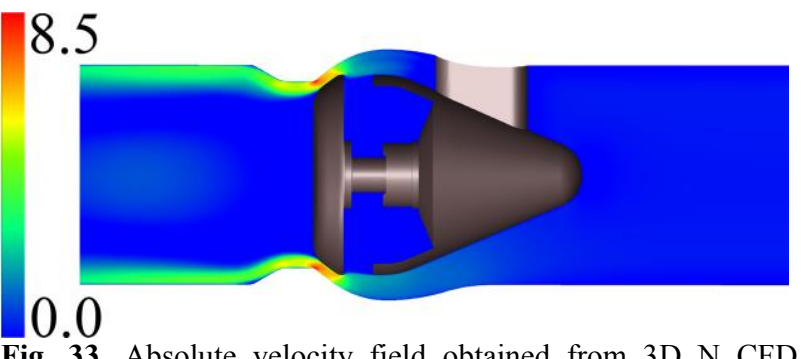

Fig. 33. Absolute velocity field obtained from 3D N CFD simulation, flow rate Q2.

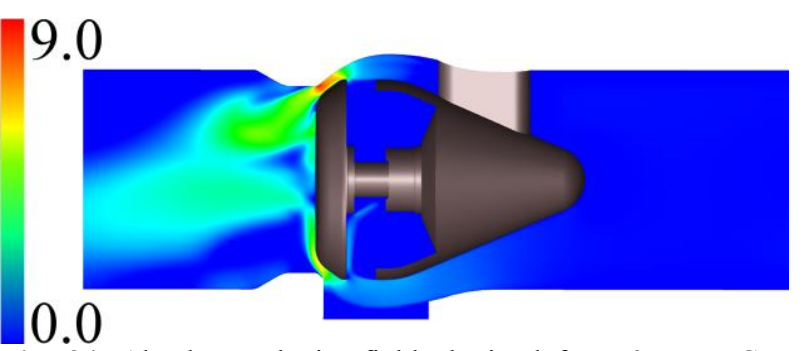

Fig. 34. Absolute velocity field obtained from 3D NP CFD simulation, flow rate Q2.

In addition to the velocity field, the field of static pressures will be plotted (Fig. 35 - 44). The same scale was retained in the figures wherever it was possible, or at least the comparative value was marked. The first is shown the pressure field for the flow direction Q1. Present pressures are relative and their real value would be obtained by adding the absolute static pressure in the pipeline system. Significant differences are not observable in addition to the pressure magnitudes before the disc in Fig. 35 and 36. The extension of the lower pressure zone behind the plug is shown in Fig. 37 . The guide disc in combination with the valve body partly behaves as a diffuser and causes a faster pressure increase in the guide disc area (Figs. 35 and 36).

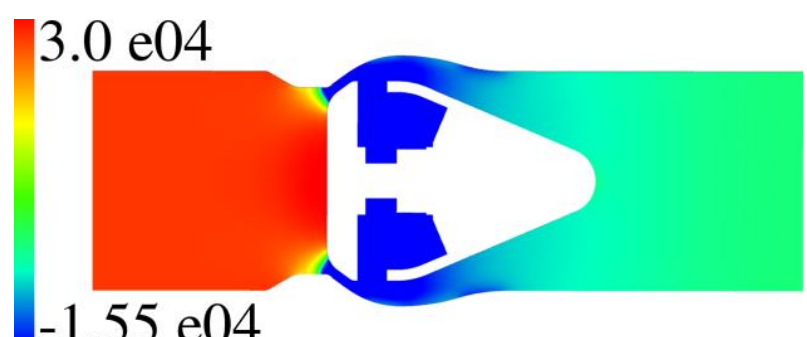

Fig. 35. Static pressure field obtained from 2D N CFD simulation, flow rate $\mathrm{Q} 1$.

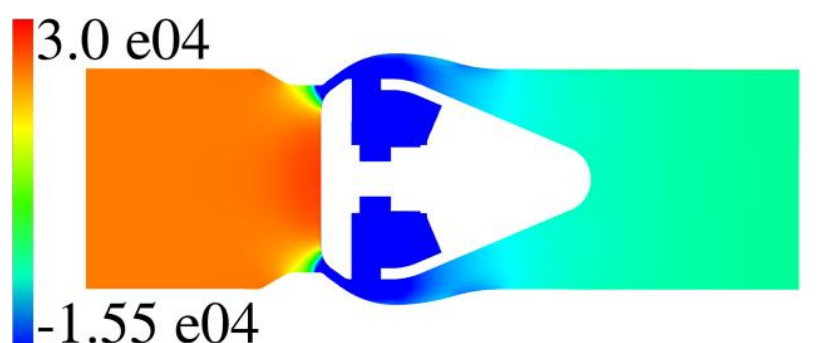

Fig. 36. Static pressure field obtained from 2D SM CFD simulation, flow rate Q1.

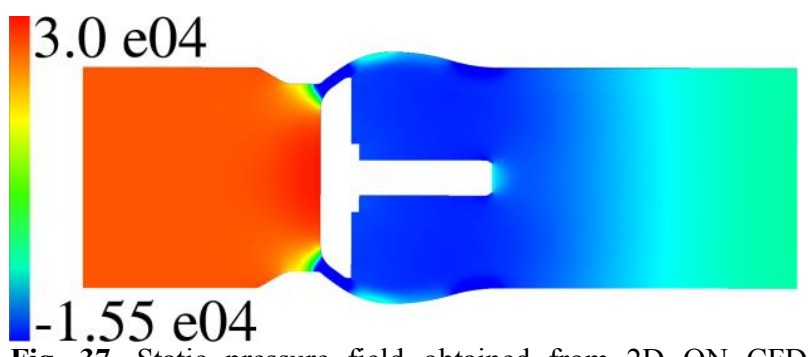

Fig. 37. Static pressure field obtained from 2D ON CFD simulation, flow rate Q1.

The lowest pressure value from the previous 2D simulations is marked on the scale map in Fig.38. Static pressure magnitudes do not disagree from the previous simulations significantly, but the pressure drop in the region between the disc seat and the disc is noticeable. The pressure extreme region is observable in the vicinity of the valve seat also in Fig. 39. The pressure values in Fig. 38 and Fig. 39 are almost identical in the area between the disc and the guide disc. The value of -1.55 E-04 in Fig. 39 is marked down on the scale map. The window at the bottom of the valve is again the most significant difference that appreciably changes the pressure distribution around the seat of the disc. The increase in static pressure in front of the valve disc compared to the valve without the windows is also noticeable. The expected increase in static pressure around the valve fin is evident.

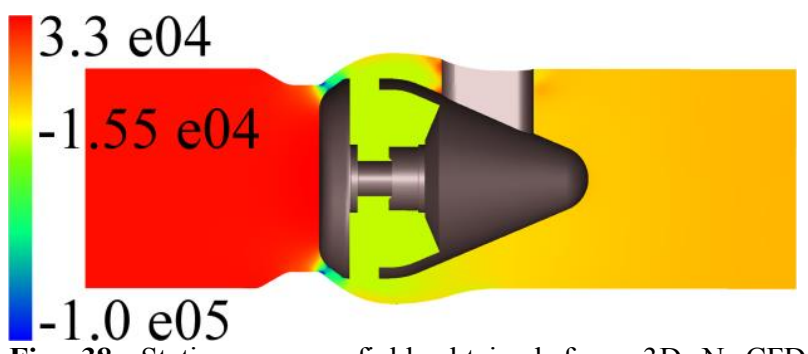

Fig. 38. Static pressure field obtained from 3D N CFD simulation, flow rate $\mathrm{Q} 1$.

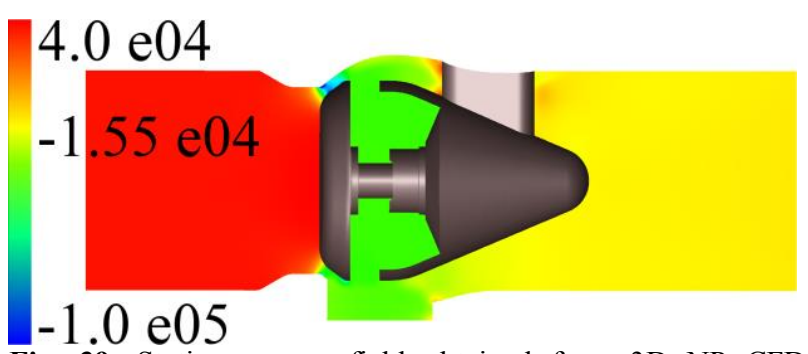

Fig. 39. Static pressure field obtained from 3D NP CFD simulation, flow rate Q1.

The remaining figures of the pressure fields (Figures 40 - 44) represent the upstream flow rate Q2 through the valve. Again, it was only possible to keep the same scale map on 2D simulations. In comparison with $2 \mathrm{D}$ and $3 \mathrm{D}$ simulations (Figures 40, 43 and 44), the difference between the highest and lowest values of the static pressure is approximately equivalent to the variants $2 \mathrm{D} \mathrm{N}$ and $3 \mathrm{D} \mathrm{N}$. However, the static pressure distribution is different. This situation has already been mentioned in the description of the velocity field behind the valve plug in the direction of flow in Fig. 30. It is again possible to see the essential differences in the 3D NP geometry in Fig. 
44. Extreme pressure values vary, and the viewports create remarkable asymmetry of the pressure field.

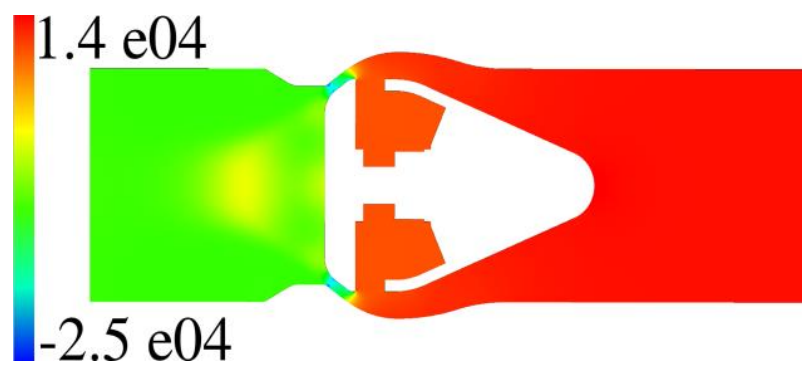

Fig. 40. Static pressure field obtained from 2D N CFD simulation, flow rate Q2.

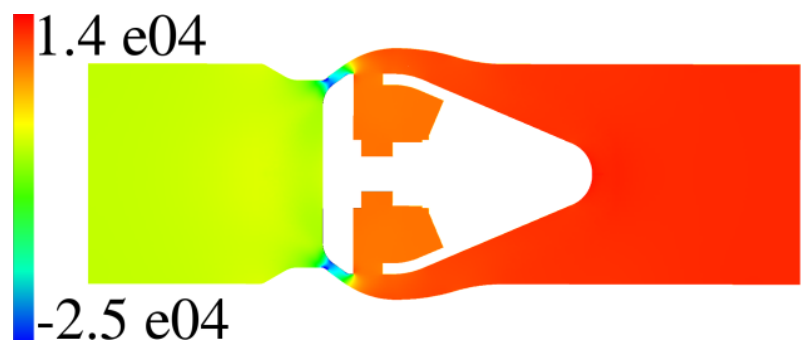

Fig. 41. Static pressure field obtained from 2D SM CFD simulation, flow rate Q2.

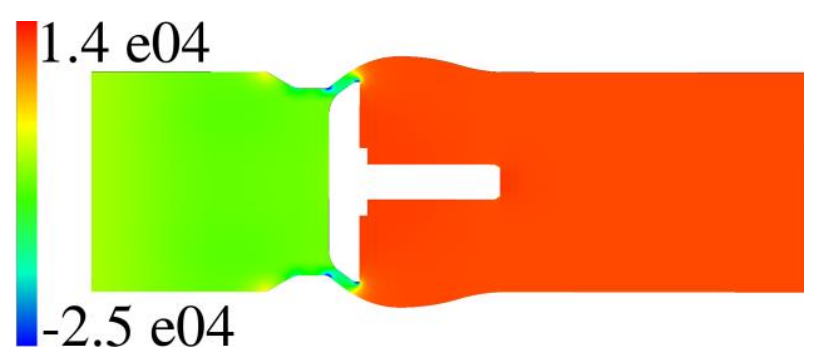

Fig. 42. Static pressure field obtained from 2D ON CFD simulation, flow rate Q2.

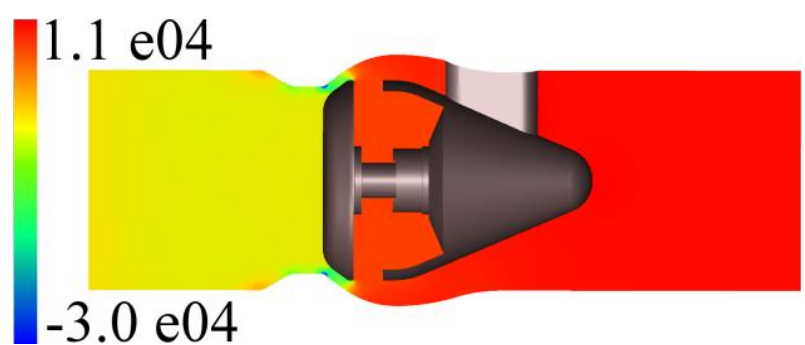

Fig. 43. Static pressure field obtained from 3D N CFD simulation, flow rate Q2.

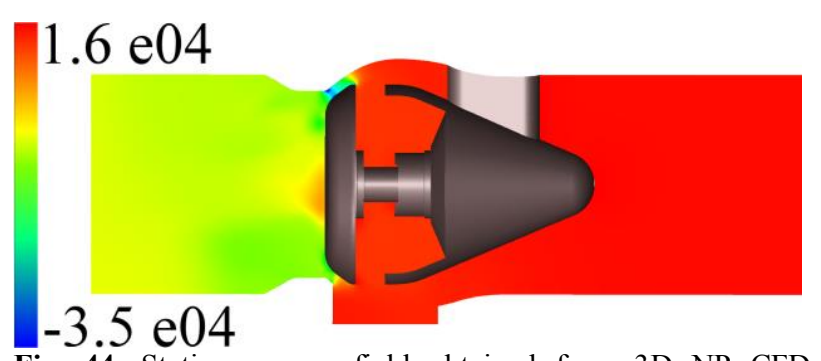

Fig. 44. Static pressure field obtained from 3D NP CFD simulation, flow rate Q2.

\section{Conclusions}

The presented study was intended as an information supplement that could not be directly obtained from the experimental testing and which should to serve as a basis for further simulation of dynamic valve behavior. An experiment was realized a little later for verification purposes. Another task was to monitor and assess possible simplifications with regard to 2D CFD simulation, onedimensional valve analysis and computational requirements.

The first question that dealt with was the general setting of CFD simulations and the choice of appropriate wall functions. Non-equilibrium wall function and scalable wall function were selected for comparison. Nonequilibrium wall function was evaluated as more accurate for 3D geometry after confrontation with experimental data,. The RNG $\mathrm{k}-\varepsilon$ turbulence model recommended in the literature did not achieve better results.

One of the experimental devices was equipped with windows to better understand the operation of the valve. The windows should be important especially for real-time analysis of the velocity field and also for basic evaluation of the disc movement depending on flow rate through the valve. Two 3D geometries with and without windows were created for this purpose. It was possible in advance to anticipate that the windows would be reflected in the static characteristics of the valve. The deformation of the velocity and pressure fields around the window frames, which is manifested in the magnitude of forces acting on the disc, is more important in this respect. Therefore, it is shown that the analysis of dynamic properties on the experimental valve with visors is not simply transferable to real device. Of course, the analysis is rather complicated as it is difficult to build a one-dimensional mathematical model and predict the valve motion for different flow rates.

There is a very good agreement visual qualitative comparison of the velocity and pressure fields in the operating flow direction. Of course, results from 2D and 3D axisymmetric geometries are compared. The 2D geometry fails in the backflow, and especially the velocity field varies considerably. The applicability of 2D simulations is therefore problematic with this valve. A more important comparison of $2 \mathrm{D}$ and $3 \mathrm{D}$ is apparent in static valve characteristics. It could be said that both geometries differ only by the embedded fin, yet this fin contributes to the deformation of the loss and flow coefficient characteristics. It is usual that the loss coefficient with a higher flow rate decreases and the flow coefficient increases on the contrary. However, the trend of both coefficients in 3D geometry changes to higher flow rates and the loss coefficient increases and the flow coefficient decreases. The described effect was not apparent in 2D geometry, despite the revision of the computational mesh. It can be said that the $2 \mathrm{D}$ and $3 \mathrm{D}$ simulations correspond to velocities of up to $6 \mathrm{~m} \cdot \mathrm{s}^{-1}$.

The possibilities of building a one-dimensional mathematical model describing the valve disc movement depending on the flow rate in the case of two-dimensional simulations were also tested. The force effects on the disc can be described by force acting on the remaining surfaces of the valve definition volume, as can be seen from the definition of force on the disc or directly from the integration of the Navier-Stokes equation. However, it is 
necessary to know the differences in static pressure within the valve. For this reason, CFD simulation of the slip valve body and CFD simulation of the valve body equipped with the plug itself was created. The ratio of the loss coefficients of the valve to the non-friction body valves is quite different. This ratio is approximately 90 $95 \%$ for full valve opening, and drops to $65-70 \%$ when the disc is evidently closer to the valve seat. This fact should be taken into consideration in a one-dimensional model if only the aggregate data from the experimental testing is available. The most unfavorable situation occurs in the case of the analytical model of the unsteady force component of the fluid acting on the valve disc. The CFD simulation shows that it significantly differs from the prediction of the analytical model. This difference increases with the shut-off time of the valve and has its extreme corresponding to a specific flow rate. The best agreement of the analytical model with numerical arrives at the normal piping system unrealistic shut-off time of the valve $\boldsymbol{T} \boldsymbol{s}=0.2 \mathrm{~s}$. An analytical model of unsteady force at this $\boldsymbol{T} \boldsymbol{s}$ value achieves $40-80 \%$ predictions from CFD simulations.

This work has been supported by Technology Agency of the Czech Republic under the project Innovative research of check valves for extreme operating conditions in energetics TH01011352.

\section{References}

1. V. J. Sonawane, T. J. Rane, A. D. Monde, R. V. Vajarinkar, P. C. Gawade, Design and Analysis of Globe Valve as Control Valve Using CFD Software, IOSR J. of Mech. and Civil Eng. pp. 63 - 71 (2013)

2. P. Kurian, C. R. Krishnamurthy, R. Rajesh, Int. J. of Emerging Tech. and Adv. Eng. 4, 10 pp. 391-397 (2014)

3. D. C. Wilcox, Turbulence Modeling for CFD (DCW Industries, Incorporated, 1994)

4. V. C. Patel, W. Rodi, G. Scheuerer, Turbulence models for near-wall and low Reynolds numbers flows: A review, AIAA J. 23(9), pp.1308-1319 (1985)

5. J. Bredberg, Internal Report 00, 4, (Department of Thermo and Fuid Dynamics, Göteborg, Sweden, 2000)

6. M. Chmielewski, CMST 19(2), pp. 107-114 (2013)

7. K. Hanjali'c, B.E. Lauder, J. Fluid Mech. 74, pp. 593610 (1976)

8. S. Sibilla, M. Gallati, J. Fluids Eng. 130, 121101 (2008)

9. Q. Yang, Z. Zhang, M. Liu, J. Hu, Procedia Eng. 23, p. 547 (2011)

10. A. Montorfano, F. Piscaglia, G. Ferrari, Mathematical and Computer Modelling. 57, 7-8, pp. 1640-1647 (2013)

11. J. Nordström, Well Posed Problems and Boundary Conditions in Computational Fluid Dynamics (22nd AIAA Computational Fluid Dynamics Conference, Dallas, 2015)

12. J. A. Valverde, S. H. Frankel, G. P. Salvador, Threedimensional control valve with complex geometry: CFD modelling and experimental validation (34th
AIAA Fluid Dynamics Conference and Exhibit, Portland, Oregon, 2004) 